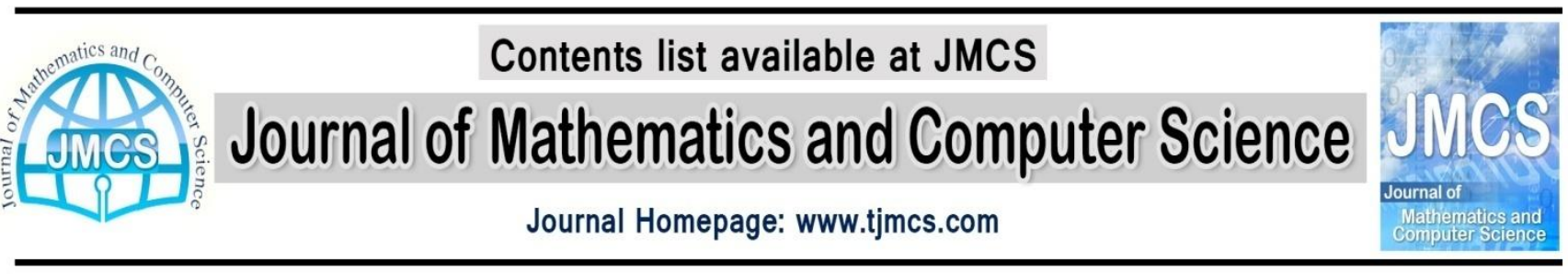

\title{
A Note on Generalization of Classical Jensen's Inequality
}

\author{
P.O. Olanipekun, A. Mogbademu \\ Departmentof Mathematics, Universityof Lagos, Yaba-Nigeria \\ peter.olanipekun1@students.unilag.edu.ng, amogbademu@unilag.edu.ng
}

Article history:

Received August 2014

Accepted September 2014

Available online September 2014

\section{Abstract}

In this note, we prove a new generalisation of the Jensen's inequality by using a Riemann-Stieltjes integrable function and convex functions under a mild condition. An example was given to support the claims of this paper.

Keywords: Convex functions, Jensen's inequality.

\section{Introduction and Preliminaries}

In [5], Royden and Fitzpatrick, examined the classical form of Jensen's inequality [3]

$$
\varphi\left(\int_{0}^{1} f(x) d x\right) \leq \int_{0}^{1}(\varphi o f)(x) d x
$$

Using the notion of the supporting line that exists at the point $(\alpha, \varphi(\alpha))$ for the graph of $\varphi$ where $\alpha \in(0,1)$. Indeed, they gave a short proof for the Jensen's inequality. The purpose of this paper is to employ a simple analytic technique which is independent of the idea in [4] to show that for any two convex functions $\varphi(x), \beta(x)$ and another Riemann Stieltjes Integrable function $f(x)$ defined on $[a, b]$ then

$$
\varphi\left(\int_{a}^{b} f d \beta\right) \leq \int_{a}^{b} \varphi(f) d \beta
$$

under a mild condition. 
Remark: A case where $\beta(x)$ is the identity function and $b-a=1$ gives the kind of Jensen's inequality discussed in [5].

The following well known definition and Lemmas are useful in the proof of our results.

Definition 1.1. A function $\varphi$ is convex on $[a, b]$ if, $\varphi(x) \leq \varphi(y)+\frac{\varphi(t)-\varphi(y)}{t-y}(x-y)$, where $a \leq y \leq x \leq t \leq b$.

Lemma $1.1([1,2][5])$. Suppose $\varphi$ is convex on $[a, b]$ and differentiable at $\alpha \in(a, b)$, then,

$$
\varphi(\alpha)+\varphi^{\prime}(\alpha)(x-\alpha) \leq \varphi(x), \forall x \in[\mathrm{a}, \mathrm{b}] .
$$

Proof: See Lemma 1 of [2] and Theorem 18 in Chapter 6 of [5].

Lemma 1.2 [3]. Let $\varphi$ be an increasing function on the closed bounded interval $[a, b]$, then $\varphi^{\prime}$ is integrable over $[\mathrm{a}, \mathrm{b}]$ and $\int_{a}^{b} \varphi^{\prime} \leq \varphi(b)-\varphi(a)$.

Proof: See Corollary 4 in section 6.2 of [5].

\section{Main results}

Theorem 2.1. Let $\varphi(x), \beta(x)$ be convex functions on $(-\infty, \infty)$ and $f(x)$ Riemann-Stieltjes integrable w.r.t $\beta(x)$ over $[a, b]$ such that $\beta(b)-\beta(a)=1$. Then,

$\varphi\left(\int_{a}^{b} f(x) d \beta\right) \leq \int_{a}^{b}(\varphi o f)(x) d \beta$.

Proof. Let $\alpha=\int_{a}^{b} f d \beta$.

Choose $m \in \mathbb{R} \ni y=m(t-\alpha)+\varphi(\alpha)$ is the equation of the supporting line passing through $(\alpha, \varphi(\alpha))$ for the graph of $\varphi$. Clearly, $\varphi^{\prime}\left(\alpha^{-}\right)<m<\varphi^{\prime}\left(\varphi^{+}\right)$. From Lemma 1. 1, we have:

$$
\varphi(t) \geq m(t-\alpha)+\varphi(\alpha) \forall t \in \mathbb{R}
$$

And, in particular

$$
\varphi(f(x)) \geq m[f(x)-\alpha]+\varphi(\alpha) \text { for } x \in[a, b](2.3)
$$

Integrating both sides of (2.3)

$$
\begin{gathered}
\int_{a}^{b} \varphi(f(x)) d \beta \geq \int_{a}^{b}(m[f(x)-\alpha]+\varphi(\alpha)) d \beta \\
=m \int_{a}^{b} f(x) d \beta-m \alpha[\beta(b)-\beta(a)]+\varphi(\alpha)[\beta(b)-\beta(a)]
\end{gathered}
$$




$$
=m \alpha-m \alpha+\varphi(\alpha)
$$

$=\varphi\left(\int_{a}^{b} f d \beta\right)$.

That is, $\int_{a}^{b}(\varphi \circ f) d \beta \geq \varphi\left(\int_{a}^{b} f(x) d \beta\right)$ completing the Proof.

\section{Example}

Let $\beta(x)=\left\{\begin{array}{lr}0, & x=a \\ 1, & a<x \leq b\end{array}\right.$

Clearly, $\beta(b)-\beta(a)=1$ and for any convex function $\varphi$ and Riemann Integrable function $f$ on [a, b], then Theorem 2.1 holds.

Acknowlegement: The authors wish to acknowledge the warm support of the Research Group in Mathematics and Applications of the University of Lagos, Nigeria.

\section{References}

[1] H. ASNAASHARI, Some remarks on convexity of Chebysev sets, The Journal of Mathematics and Computer Science, Vol. 1 No. 2 (2010) 102-106.

[2] H. FINBARR, A note on the Mean Value Theorem, https://cms.math.ca/crux/v22/n7page 290293.pdf

[3] R. Kargar, A. Bilavi, S. Abdolahi, S. Maroufi, A class of Multivalent analytic functions defined by a new linear operator. Journal of Mathematics and Computer Science, 8(2014) 236-334.

[4] D. S. MITRINOVIC, J. E. PECARIC, and A. M. FINK, Classical and New Inequalities in Analysis, Kluwer Academic Publishers, London, 1993.

[5] H. L. ROYDEN, and P. M. FITZPATRICK, Real Analysis, Fourth Edition, PHI- Learning Private Limited, New Delhi, 2011. 